\title{
Artikel
}

Peter Neumann*

\section{Der Deutsche in der Landschaft - Borchardt und Benjamin}


Abstract: Im Ausgang von Walter Benjamins methodologischer Kritik an Rudolf Borchardts Anthologie Der Deutsche in der Landschaft zeigt der Aufsatz, in welchem Maße der jeweilige Zugriff auf ,Geistesgeschichte' mit einem spezifischen Verständnis von Natur und, geistiger Zugehörigkeit' verbunden ist. Führt die Einheitlichkeit der Naturbetrachtung nach Goethe'schem Vorbild bei Borchardt zu einem Verständnis von Geschichte als epochenübergreifender Kontinuität und wiederzugewinnender Tradition, bricht bei Benjamin unter dem Vorzeichen des Faschismus das Anschauungskontinuum organisch gewachsener Kulturalität zusammen - ,Heimat' wird in natürlicher wie in geistiger Hinsicht prekär. Das ,erfahrungssatte Dasein in der Landschaft' ist nurmehr im Modus des ,Eingedenkens' möglich, als Zeugnis seines eigenen Untergangs.

Taking its cue from Walter Benjamin's methodological critique of Rudolf Borchardt's anthology Der Deutsche in der Landschaft, the article demonstrates how these different approaches connect 'intellectual history' and a specific idea of nature. While Borchardt proposes a unifying vision of nature (modelled after Goethe's) that leads to a concept of history as continuity and a tradition that ist to be regained; with Benjamin the idea of a conceptual continuum and organic culturality collapses in the wake of fascism: 'Heimat' becomes precarious intellectually and naturally. Being-in-landscape as a saturated experience is furthermore only possible in a mode of remembrance, that is, as a testament to its own demise.

Keywords: Natur, Landschaft, geschichtliche Identität, Diskontinuität, Morphologie

*Dr. Peter Neumann, Carl von Ossietzky Universität Oldenburg, Institut für Philosophie, E-Mail: Peter.Neumann@uol.de

„Es ist der Deutsche in der Länderwelt der Erde, der Deutsche in der Landschaft, und es ist darum ein nur innerhalb der deutschen geistigen Geschichte und Charakterwelt, nur deutsch mögliches Buch", heißt es bei Rudolf Borchardt im Nachwort zu seiner erstmals 1927 in der Bremer Presse veröffentlichten Sammlung deutscher Landschaftsbetrachtungen, deren Titel - Der Deutsche in der Landschaft - heute unfreiwillig komisch klingen mag, ein Jahrzehnt nach dem Ende des Ersten Weltkriegs, nach dem so schmachvoll empfundenen Waffenstillstandsabkommen von Compègnie aber alles andere als humorvoll gemeint war: „Ein Engländer in der Landschaft, ein Franzose, ein Italiener, ein Holländer, ein Russe in der Landschaft ist ihm [dem Deutschen, P.N.] von keiner Seite außerhalb unserer Grenzen her zur Seite zu stellen" (Borchardt [1927] 2018, 495). ${ }^{1}$ Vor dem Hintergrund dieser unverhohlenen, für die Zeit aber durchaus einschlägigen Äußerung nationalreaktionärer Gesinnung ist es umso verblüffender, dass gerade ein in geistesgeschicht-

1 Matthes \& Seitz hat das Werk kürzlich erst wieder aufgelegt und mit einem Nachwort von Franck Hofmann versehen. lichen Fragen so beschlagener und in der ästhetischen Moderne Charles Baudelaires beheimateter Denker wie Walter Benjamin in den höchsten Tönen von der Landschaftsanthologie Borchardts schwärmt und damit offenkundig auch deren implizite geschichtsmethodologischen Voraussetzungen teilt: Der Titel, so Benjamin in einer erstmals 1928 in der Literarischen Welt erschienenen Rezension, sei mehr als eine "glückliche Fügung": "Eine Entdeckung und die Hoffnung seines Herausgebers, mit inm ein Stück, verlorener deutscher Geistesgröße' einzubringen, wird jeder Leser an sich erfüllt finden" (GS III, 93).

Benjamins Urteil über Borchardts Sammlung muss auch deshalb überraschen, weil es die Brücke zu einer Anthologie schlägt, die er acht Jahren später selber herausgeben sollte und die allein schon in der Wahl des Titels, Wahlverwandtschaften' erkennen lässt, die der näheren Erläuterung bedürfen: Deutsche Menschen. Eine Folge von Briefen, 1936 im Züricher Verlag Vita Nova erschienen; Benjamin lebte damals schon in Paris, im Exil, und hatte sich entschieden, das Buch unter Pseudonym veröffentlichen zu lassen: Detlef Holz, ein dumpfdeutscher Name, dumpf genug, um das Buch an den deutschen Behörden 
vorbei ins Land zu schleusen. Auch Benjamin geht es in seiner Briefanthologie - nicht anders als Borchardt - um ,verlorene deutsche Geistesgröße'. Die Geschichte des 19. Jahrhunderts und damit die Vorgeschichte derjenigen Katastrophe, die mit der "Machtergreifung" der Nationalsozialisten sich endgültig zu der Katastrophe auswachsen sollte, die später auch folgte, deutet Benjamin als eine Verfallsgeschichte, in der das Bürgertum sich zwar emanzipierte und zur politischen Kraft aufstieg, vom Geist, der es ursprünglich trug, aber nur die Posten, Titel und Ämter am Ende übriggeblieben. In diesem Gestus der ,Krise' nähert sich Benjamin Borchardts Schablone an. Nur zielt die Anlage seiner Sammlung methodisch in eine ganz andere Richtung, was den Fall nun endgültig zum Rätsel werden lässt (Vgl. Benjamin 1936).

Während Borchardt ganz und gar auf die "Restitution verlorener deutscher Geistesgröße" (Borchardt [1927] 2018, 507) im Sinne einer schöpferischen Restauration der durch den vermeintlichen Bruch im 19. Jahrhundert offenbar verloren gegangenen Tradition abendländischen, und das heißt: in erster Linie deutschen Denkens setzt, geht es Benjamin darum, im Moment des Untergangs, die geschichtlichen Kräfte heraufzubeschwören, die, obwohl sie zwar gerade diesen Untergang nicht verhindern konnten, ihn in ihrer Wirkungslosigkeit vielmehr gerade mit ermöglicht haben, noch immer ein Alteritätspotenzial in sich bergen, Zeugnis der Hoffnung auf eine Geschichte sind, die auch hätte, anders' verlaufen können. Hier die Konstruktion geschichtlicher Kontinuität, dort Konstellationen geschichtlicher Diskontinuität. In Borchardts deutschem Kulturnationalismus und Benjamins messianischem Geschichtspessimismus liegt, mit anderen Worten, "das ganze Katastrophenbewußtsein der Epoche und ihre Erwartung" beschlossen. (Matz 2011, 82f.; Vgl. Haas 1928) Entgegengesetzter könnten Methodologien ,deutscher Geistesgeschichte' kaum ausfallen.

Wie also ist die Diskrepanz zwischen der Wertschätzung für Borchardts Landschaftsanthologie auf der einen Seite und der entgegengesetzten methodischen Ausrichtung von Benjamins eigener Briefanthologie auf der anderen Seite zu verstehen? Und warum lässt sich aus dieser Spannung heraus, der Konstellation BorchardtBenjamin, ein Beitrag zur Konfliktgeschichte ,prekärer Heimat' formulieren? Die These, die es im Folgenden zu entfalten gilt, lautet: Die Art und
Weise, wie Borchardt und Benjamin ihren Zugriff auf ,deutsche Geistesgeschichte' jeweils methodisch gestalten, ist aufs Engste mit ihren Konzepten von Natur und Heimat als, geistiger Zugehörigkeit' verbunden, die, wie sich zeigen wird, nicht nur verschiedenartig ausfallen, sondern in der Polarität ihrer Anlage eine Spannung in die fragliche Zeit um 1900 eintragen. 'Natur' wird dabei zum Prüfstand einer universalgeschichtlichen Zeitdiagnostik, die erst in der Reflexion auf das Allernächste, die Landschaft, die in Wahrheit aber auf das Allerfernste, das geschichtliche Telos des Geistes, verweist, zu ihrer eigenen Leitbegriffen und Grundsätzen gelangt.

\section{Geistige Landschaften}

Borchardts Auffassung von Geistesgeschichte ist von Grund auf geprägt vom Festhalten an Tradition, vom Glauben an eine epochenübergreifende geschichtliche Kontinuität. Dieses Kontinuitätsaxiom bildet bereits den Grundtenor eines seiner frühesten Werke, des Essays Villa, in dem er sich mit der Typologie eben dieser spezifisch italienischen Baugattung, der des Landhauses, auseinandersetzt und dabei auf sprachlich wie theoretisch hochvirtuose Weise zur Einsicht gelangt, dass Landschaft und Geschichte eins seien, ja, dass die Villa geradezu Symbol einer Einheit sei, in der sich Natur und Kultur durchdringen. ${ }^{2}$ Schon das Motto - ein freies Zitat aus Ciceros De finibus bonorum et malorum - steckt den gedanklichen Kosmos ab, in dem sich Borchardt bewegt und den er zeitlebens auch nicht mehr verlässt: ,Wohin wir auch gehen, wir setzen den Fuß in Geschichte'. ${ }^{3}$

In der Landschaftsanthologie macht sich das Kontinuitätsaxiom auf der kompositorischen Ebene bemerkbar: Denn , die' Geschichte, in die da der Fuß gesetzt wird, ist niemals das rohe, unbeherrschte, hereinbrechende Chaos geschichtlich konfligierender Kräfte. Sie ist vielmehr die immer

2 Der Essay Villa erschien erstmals in zwei Lieferungen in der Frankfurter Zeitung, 51. Jg., Nr. 46 und 47 vom 15. und 16. Februar 1907, jeweils Erstes Morgenblatt S. 1-3. Im darauffolgenden Jahr kam er als Privatdruck bei Haberland in Leipzig heraus. Vgl. dazu auch Borchardt [1907] 1920. 3 Im von Borchardt verwendeten lateinischen Original lautet das Zitat: "Quocumque ingredimur, in aliquam historiam pedem ponimus". 
schon geformte, vom Geist durchdrungene, im idealistischen Sinne auf den Begriff gebrachte Wirklichkeit, weshalb sich die Frage nach der Anlage der Anthologie für Borchardt beinahe von selbst erledigt. Auf der Ebene des Geistes hat das metaphysische Prinzip organischer Ganzheiten seinen Dienst immer schon getan: „Ein Ganzes aber ist es [...], ein Ganzes und nicht ein loser Haufen trockener Staub [...], ein Ganzes, als geistiger Körper in Relation zu einem ersten andern Ganzen und einem zweiten andern Ganzen, dem Ganzen der deutschen Nation und dem Ganzen der Erde" (Borchardt [1927] 2018, 493). ${ }^{4}$ Als "Anthologie[] im höchsten Sinne" wird Benjamin die Zusammenstellung in seiner Rezension später bezeichnen und auch nicht verlegen sein, sein Urteil mit einer organizistischen Metapher buchstäblich zu ,bekränzen': "Kränze wie der des Meleager von Gadara, den wir, ob wir auch alle seine Blüten beim Namen nennen, uns nicht mehr aufgelöst zu denken wüßten" (GS III, 92).

Borchardts eigenes Stichwort lautet in diesem Zusammenhang "geistige Geographie" (Borchardt [1927] 2018, 495); er ist der festen Überzeugung, dass sich der Geist in einem und durch ein Volk bevorzugt ausspricht: dem deutschen. Sicher, es habe von Marco Polo über James Cook bis zu Pierre Louis Maupertius reiche Reisenliteraturen gegeben, gegen die lange Zeit nichts Deutsches aufzubieten gewesen sei. England, Frankreich, Italien: "Aber was will das gegen die Fülle besagen, die wir haben zusammenbringen und vorlegen können" (Borchardt [1927] 2018, 495). Nur der Deutsche habe das Vermögen, auf Höhe des Geistes zu kommen, mit anderen Worten, den "ganzen Kreis der Schöpfung" auszuschreiten, indem er das durch Rousseau befreite, pathetisch und kosmisch gewordene Naturgefüh/ auf eine höhere Stufe der Form zu heben verstehe, ohne ihm dadurch etwas von seiner Tiefe und Emphase zu nehmen, kurz: indem er das Niederste zum Höchsten mache:

Was haben die alten Reiseliteraturen der beglückten Völker zur Begründung plastischer Erdansicht, naturwissenschaftlicher Durcharbeitung der Voraussetzung zu geographischer Begriffsbildung, zu geistiger Geo-

4 Eine analoge gedankliche Bewegung lässt sich auch in Pisa. Ein Versuch von 1938 beobachten, in dem Borchardt die einzelnen Kunstwerke stets mit Hilfe eines verbindenden Allgemeinen aufeinander bezieht. Vgl. dazu Steiger 2004, S. 32. graphie, zur Zusammendenkung von Struktur des anorganischen und Decke des organischen Stoffes, und zu all dem unzähligen beigetragen, worin dies Buch, das zwar auch Reisestücke enthält, zwar auch unvergessliche Tropenbilder, aber daneben die Seiten Goethes und Heinses, Träume neben Vesten, Gedichte neben erhabenen Verallgemeinerungen, - worin also es allerdings den ganzen Kreis der Schöpfung ausschreitet. (Borchardt [1927] 2018, 495f.)

Der Deutsche ist nach Borchardt ein nie zur Ruhe gekommenes "Kind der Völkerwanderung", der immer wieder in die Welt hinausgeht, aus der Bücherstube hinaus, und sie mit dem Geist erobert, sei es - und hier folgt ein Formenkanon ,geistiger Geographie' - als (1) sinnlich empfindendes und darstellendes Verhältnis zur Landschaft, (2) als geologisch-geognostische Perspektive auf Struktur, (3) als naturwissenschaftlich ordnender Standpunkt zum Kosmos, (4) als im strengen Sinne geographische Ansicht der Erde, (5) als historisch-geografische Einstellung zur Nation. Ganz gleich: Der Deutsche ist "überall zu Haus und nicht zu Haus”, er ist „zu Haus wo er eben steht”: „Die Welt geht in inn ein, indes er in die Welt aufgeht. Er ist der alte Wanderer seiner Geschichte, der Gast auf Erden" (Borchardt [1927] 2018, 496).

Was bei Borchardt auf diese Weise geistesgeschichtlich zusammenfindet, ist eine idealistisch grundierte Geschichtsphilosophie und der um 1900 wiederaufflammende Mythos von der deutschen Kulturnation - ein Amalgam, das als verkapptes, säkularisiertes Eschaton vor keiner Grenze Halt macht und über kurz oder lang sich sein aufgespartes geschichtliches Recht zu verschaffen weiß. Nicht deutsche Landschaften stehen deshalb im Zentrum der Anthologie, sondern deutsch angesehene Landschaften: von Johann Georg Sulzers Gartenlandschaften zwischen Antibes und Nizza über Karl Philipp Moritz Beschreibung der Villa Borghese bis hin zu Anton Prokesh von Ostens Orient-Studien von Alexandria bis Salamis. Die Anthologie ist bewusst gerahmt von Ansichten nicht-deutscher, teils sogar nicht-europäischer Landschaften, die in ihrer natürlichen, aber auch kulturellen Gestalt unterschiedlicher nicht sein könnten. Dazwischen Haiti, Java, die Anden, Japan, Kaschmir, der Ararat, die Pyrenäen, Griechenland, Italien, immer wieder Italien.

Als ,deutscher Universalismus' lässt sich ein solcher Geschichtschiliasmus schon um 1800 beobachten, als durch die Französische Revolu- 
tion die Identitäten flüssig zu werden beginnen und auch das bevorstehende Ende des Heiligen Römischen Reiches Deutscher Nation Fragen der kulturellen Dominanz und nationalen Zugehörigkeit in einem neuen Licht erscheinen lässt. Und wie damals kreuzen sich auch um 1900 zwei sehr unterschiedliche Geschichtsbestrebungen: der Kosmopolitismus auf der einen und der Nationalismus auf der anderen Seite, eine kulturelle Antinomie, die vor Augen führt, dass in Wahrheit nicht, wie von Vertretern und Kronzeugen dieser Position behauptet, die Entfaltung einer über das Deutsche hinausreichenden Kultur des Humanum, sondern die Bildung einer ganz bestimmten Nation Endzweck der geistigen Bestrebungen ist, die sich natürlicherweise auch mit ganz bestimmten Besitzverhältnissen und Machtansprüchen verbindet (Vgl. Schmidt 2004; Höfer 2015). Die Frage nach dem Deutschen in der Landschaft tritt nicht hinter die Frage nach dem einen Menschen zurück. Nicht von ungefähr positioniert sich ein deutscher Universalismus dezidiert gegen sein französisches Pendant. Das Ganze, zu dem sich alles bildet, in dessen Dienst alles zu stehen hat, ist die Nation, die deutsche, und die hat in einer Zeit bis zur gesellschaftlichen Zerrissenheit gespannter, äußerster historischer Belastung mehr denn je sich ihrer eigenen Tradition und Herkunft zu vergewissern. Heute nicht weniger als damals.

Hier, in dieser Indienstnahme, ist das geistige Zentrum eines nationalkonservativen Autors wie Rudolf Borchardt zu verorten. Denn es braucht Schriftsteller wie inn, intellektuelle Führer, die gerade diese verloren gegangene Tradition unter dem Schutt der letzten Jahre, so vieler Jahrzehnte wieder zu bergen, behutsam hervorzuholen und als zeitlos geltenden Maßstab zu vermitteln wissen, um der deutschen Nation das unverwechselbare Profil zu geben, das sie zu keinem Zeitpunkt der Geschichte je hatte. Im Falle von Borchardt kann man sogar davon ausgehen, dass es um mehr als eine Form bloßer Vermittlung geht. Es geht um das spekulative Prinzip geistiger Verkörperung: Für die Erneuerung der Nation braucht es Leitfiguren wie inn, Borchardt, Personen, in denen die deutsche Tradition überdauert hat, die selber ,deutschen Menschheitsgeist' verkörpern (Vgl. Kauffmann 2003).

In die Zeit der Landschaftsanthologie fällt auch eine Rede, deren Titel zum programmatischen Schlagwort für Borchardts Denken insgesamt geworden ist: Schöpferische Restauration. In der Rede setzt Borchardt sich mit dem auseinander, was im 19. Jahrhundert so unwiderruflich in die Geschichte des Geistes hereingebrochen ist und was aus seiner Perspektive nicht sein kann, weil es nicht sein darf: der Bruch mit der historisch überlieferten Tradition abendländischen, und das heißt auch hier: deutschen Denkens, der Verfall des Geistes, die Entgötterung des Himmels, Ideenfrevel, die ideologische wie ökonomische Korruption des Volkes, die - national gewendet - in die Polarität von Deutschland und Frankreich zurückschlägt:

Seelenlos und geistlos, eine durchsichtig und gewalttätig konstruierte Abstraktion, steht der Staat Friedrich des Großen, der ihm nachgeäffte Karl Eugens, auf seine geworbenen und gepreßten Heere gestützt, den zentralisierten Gewaltstaaten jenseits des Rheins gegenüber, innen abgesehen wie die von Versailles geschorenen Schnurregelgärten mit boulingreen und porzellanenen Oreaden. Diese gesamte Welt ist käuflich und bewegt sich in einem wilden Tanze um ihre Kaufpreise. Käuflich der Staat, käuflich Amt und Ehre, Familie und Recht, Poesie und Wissenschaft, Mann und Weib und Kind. Das Schöne ist fast überall zum Eleganten, zum Hübschen, zum Niedlichen, zum Reiz, zum Anreiz, ja zu dem aus halber Abstoßung entstehenden Widerreiz entartet. (Borchardt [1927] 1955, 233)

Als Gegenmaßnahme kommt für Borchardt nur die Wiedergeburt der Nation aus dem Geist der Romantik in Frage - einer Romantik, die hier nicht als spezifischer Gegenbegriff zur Klassik auftritt, sondern das Ganze der deutschen Literatur und Philosophie an der Epochenschwelle, um 1800' meint, von Herders Einsicht in die Geschichtlichkeit der menschlichen Gattung über Georg Forster heimische wie abenteuerliche Reisebeschreibungen bis zum Kosmos, dem opus magnum des späten Alexander von Humboldt. Sie alle repräsentieren nach Borchardt auf die ein oder andere Weise den menschheitsbildenden Geist der deutschen Nation. Und es ist natürlich kein Zufall, dass er mit dieser Auffassung keineswegs allein ist, sondern in bester Gesellschaft von Literaturwissenschaftlern seiner Zeit wie Hermann August Korff, die Geistesgeschichte in vergleichbarer Weise unter das Diktat einer Idee stellen, sodass der historische Einzelfall einen zutiefst ambivalenten ontologischen Status bekommt: Entweder er wird zum Ausdruck der Idee, dann existiert er nicht mehr als Einzelfall, sondern nur noch als Symbol eines übergeordneten Allgemeinen, oder aber, nicht 
weniger problematischer, er drückt die Idee nicht aus, dann existiert er nicht, noch nicht mal als Einzelfall. Korff bezeichnet jene Form der Ideenorganisation als "Siege des Geistes über die jeweiligen Fragwürdigkeiten des Lebens" (Korff 1923, 5).

Sprechenderweise fehlt in der Sammlung denn auch ein Autor, den man unter den epochemachenden Vorzeichen einer Landschaftsanthologie wie dieser mit Sicherheit vermutet hätte. Das gilt selbst dann, wenn Borchardt als Herausgeber nach eigenen Aussagen an einer systematischen Vollständigkeit gar nicht interessiert ist - und dies methodisch auch gar nicht sein kann. Die Versicherung schützt nicht vor dem mit Blindheit geschlagenen System, das sie hervorbringt. Wenn es um das Verhältnis des Deutschen zur Landschaft geht und der Landschaft zu Deutschland, fehlt eben einer, ohne den es nicht geht: Heinrich Heine. Keines seiner ,Reisebilder' hat Eingang in die Anthologie gefunden, ,ausgeschaltet" (Borchardt [1927] 2018, 501), wie Borchardt selbst entlarvend begriffslos argumentiert: Sie, Heines ,Reisebilder', dienten nur als Vorwand, um dem Publikum die eigene "Gefallsucht" und „Rührwitzigkeit" zu präsentieren. Heine war und blieb - mit Adorno gesprochen - die "Wunde" der deutschen Literatur, auch und nicht zuletzt bei Borchardt (Vgl. Adorno 1974).

Konnte einer wie Benjamin, der über den Begriff der Kritik in der Romantik promoviert wurde und der wie kaum ein anderer Intellektueller nach dem Ersten Weltkrieg dem Deutschkonservatismus wie überhaupt jedem Kulturnationalismus ablehnend bis feindlich gegenüberstand, für solch ein Projekt gewonnen werden? Ja, anscheinend. Als eine „platonische Landschaft" preist Benjamin die Anthologie in seiner Rezension, begreift sie als eine "Wirkung des ursprünglichen Schrifttums

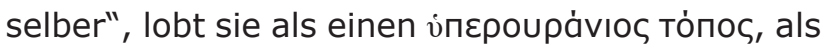
einen Ort jenseits des Himmels, in dem "anschaulich und als Urbilder Städte, Provinzen und vergessene Erdwinkel liegen" (GS III, 92f.).

\section{Idolatrie der Geschichte}

Vergegenwärtigt man sich die näheren Lebensumstände, könnte man zunächst geneigt sein, Benjamins positive Rezension auf taktisches Verhalten zurückzuführen; es wäre nicht das erste Mal, das Benjamin sein privates Urteil aus litera- turbetriebsstrategischen Gründen hinter seinem publizistischen verbirgt. Auch bei der Besprechung von Hugo von Hofmannsthals Trauerspiel Der Turm lässt Benjamin eine Skrupellosigkeit und Unanständigkeit walten, die in einem Widerspruch zu seiner ansonsten auf absoluter Bescheidenheit und Präzision beharrenden intellektuellen Redlichkeit steht. In diesem Sinne urteilt Wolfgang Matz in seiner im Ausgang von dessen Verhältnis zu Borchardt geschriebenen intellektuellen Biographie Benjamins:

\begin{abstract}
So tut man inm gewiß kein Unrecht, betrachtet man die Rezension der Landschafts-Anthologie vor allem als Angelegenheit literaturpolitischer Opportunität, - nicht gegenüber Borchardt natürlich, an dem ihm nichts gelegen war, sondern gegenüber Hofmannsthal. Benjamin war an guten Beziehungen weiterhin sehr interessiert, und seit längerer Zeit hatte er mit Hofmannsthal und der Bremer Presse den Plan entwickelt, die Schriften Wilhelm von Humboldts herauszugeben, und auch wenn der Plan nie verwirklicht wurde, so wollte Benjamin seine Verwirklichung doch mit allen Mitteln fördern. Rezensionen wie die der Anthologie oder des Turms waren da kleine, strategische und im Falle des Turms auch unaufrichtige Züge. ${ }^{5}$ (Vgl. dazu Matz 2011, 76f.)
\end{abstract}

Soweit, so plausibel. Hermeneutisch zufriedenstellend ist eine solche Analyse aber keineswegs. Um systematisches Kapital aus der Rezension zu schlagen, mag es vielmehr hilfreich sein, Benjamins Besprechung beim Wort zu nehmen, bis zu dem Punkt, an dem die Kritik, die sich zweifelsohne hinter ihr verbirgt, einsetzen müsste - und tatsächlich auch an anderer, späterer Stelle ein-

5 Auch in den Druck des Wahlverwandtschaften-Aufsatzes findet eine Borchardt betreffende kritische Aussage keinen Eingang. Zwar lässt sich nicht mehr zweifelsfrei rekonstruieren, wer den entsprechenden Satz für den Druck gestrichen hat - Hofmannsthal, in dessen "Neuen Deutschen Beiträgen" der Text erscheint, hatte sich offenbar nicht daran gestört -, aber die Skrupel, die Benjamin schon im Vorfeld begleiten haben, lassen erkennen, wie literaturbetriebsstrategisch er gedacht hat. Aufschlussreich im vorliegenden Zusammenhang ist, dass Benjamin schon hier die ,Scheinhaftigkeit' der Borchardt'schen Methodenwahl kritisiert: „Tritt in jener [sc. Hölderlins, P.N.] Lyrik das Ausdruckslose, so in Goethescher die Schönheit bis zur Grenze dessen hervor, was im Kunstwerk sich fassen läßt. Was jenseits dieser Grenze sich bewegt ist Ausgeburt des Wahnsinns in der einen, ist beschworene Erscheinung in der anderen Richtung. Und in dieser darf die deutsche Dichtung keinen Schritt über Goethe hinauswagen, ohne gnadenlos einer Scheinwelt anheimzufallen, deren lockendste Bilder Rudolf Borchardt hervorrief" (GS I.1, 182). 
setzt. Und hier fällt ein Begriff ins Auge, der die ,Dazwischenkunft eines Dritten' bedeutet, dessen Name bereits genannt wurde, und dessen anwesende Abwesenheit sich beinahe von selbst versteht: Goethe. Dessen - und nicht etwa Platons Begriff des Urbildes bildet die Folie, auf dem die Auseinandersetzung Benjamins mit Borchardts Konzeption deutscher Geistesgeschichte stattfindet: lobende Zustimmung in der Rezension einerseits, vernichtende Kritik, auf die es im Folgenden ankommt, andererseits.

Borchardts Begriff der Geistesgeschichte ist ganz von der Goethe'schen Naturauffassung durchdrungen, der Morphologie, der Lehre von der Bildung und Umbildung der organischen Körper, die sich in immer neuen, transformierten Gestalten zu erkennen geben. Dabei folgt er einer im 19. Jahrhundert einsetzenden Tradition geisteswissenschaftlicher Disziplinierung, die Goethes Anschauungsweise der Natur - das , anschauende Denken' - auf Gegenstände der Kultur und Religion, Politik und Gesellschaft überträgt. Organisch ist, was immer der Geist nach seinem Bilde empfängt, durchdringt und formt.

Nicht von ungefähr polemisiert Borchardt schon in seinem Gespräch der Formen von 1905 gegen das bloße Anhäufen von Material in der Altphilologie und in der Neugermanistik und plädiert - ganz im Sinne der Dilthey'schen Methodendifferenzierung von Natur- und Geisteswissenschaften - dafür, dass jedes gedanklich und sprachlich geformte Werk vom ,Erlebnis' auszugehen habe, dass zu einem ,geistigen Erlebnis' in der individuellen Bildungsgeschichte der Persönlichkeit werden müsse. Positivismus wie auch Historismus sind inm zuwider. Im Nachwort zur Landschaftsanthologie heißt es bei Borchardt:

Wir übergeben der Nation, da wir als Söhne des neunzehnten Jahrhunderts an die Mächte der Persönlichkeit glauben, niemals Gegenstände gegenständlich, sondern immer und immer nur Bilder der Gegenstände bildlich, nur Formen, die der Gegenstand beim Durchgang durch den organischen Geist sich umwandelnd empfangen hat, und übergeben damit, in immer neuen Abwandlungen und Anwendungen, immer neue Bilder dieses organischen Geistes selbst. (Borchardt [1927] 2018, 494)

Borchardt ist Verfechter eines Geschichtsbegriffs, dem sich alles Natürliche wie alles von Menschengeist Geformte organisch anverwan- deln lässt. Er überträgt die Goethe'sche Formprinzipien von Polarität und Steigerung auf den Bereich der Kultur und Religion, der Gesellschaft und Politik, weil gerade die geschichtliche Welt inm an allen Ecken und Enden instabil geworden zu sein scheint: pathologisch. Borchardts Landschaftsanthologie ist das Gegenbild einer in sich zerfallenen Moderne - soll es sein, nach dem Ersten Weltkrieg, mehr denn je. Hier befindet sich der Glutkern seiner geistigen Existenz als Autor und Herausgeber. Und hierin kommt auch Benjamin mit ihm überein, weswegen die positive Rezension eben nicht nur auf taktisches Verhalten reduziert werden kann. Benjamin lobt den einheitlichen Charakter der Sammlung nicht zuletzt deshalb, weil er selber der Auffassung ist, dass die in solchen Fällen „übliche[] Blütenlese" immer das "Odium der Plünderung, der unbefugten Ausbeutung" (Benjamin 1927) mit sich führt, aus der nur die Oberflächlichkeit der Zeit, die Verrohung der Sitten, die Enthumanisierung des Denkens sprechen kann, ihre Verlassenheit. Benjamin schätzt an Borchardts Sammlung, dass die Geschichte aus jedem einzelnen Beitrag spricht und das Werk dadurch in der geschichtlichen Gegenwart fortgeschrieben wird: Sie ist ein ,Eingedenken' an ein erfahrungs- und anschauungsgesättigtes Erzählen, das mit den Erfahrungen aus den Schützengräben des Ersten Weltkrieges unwiderruflich zu Schaden gekommen ist: In den von Borchardt gesammelten Texten des 19. Jahrhunderts ist die Reflexion über Sprache und Landschaft noch ,durchaus heil', wenn auch nicht bei sich oder gar zu Hause. (Vgl. Hofmann 2018, 522)

Der restlos für die Sache eingenommene Fürsprecher, als der er sich in der Rezension präsentiert, ist Benjamin aber nicht. Während Borchardt die Idolatrie der Natur, die man Goethe immer fälschlicherweise nachgesagt hat - erinnert sei an das Tobler-Fragment -, ungedeckt aufnimmt, sie auf die Kultur überträgt, sodass sie zu einer Idolatrie der Geschichte überhöht wird, favorisiert Benjamin ein ganz anderes Modell, was im Folgenden an der Anlage seiner eigenen, neun Jahre später erschienenen Anthologien, aber auch an späteren kritischen Bezugnahmen auf Borchardt deutlich werden soll. Ein erster Hinweis darauf lässt sich bereits der Rezension der Landschaftsanthologie selber entnehmen, wenn man die Codes der Benjamin'schen Begriffsverwendung 
zu entschlüsseln weiß: Benjamin konfrontiert die Fülle der von Borchardt besorgten Landschaftsbetrachtungen, deren "erfahrungssatte[s] Dasein in der Landschaft", mit der existenziellen Not, aus der sie entstanden sind. Es ist der "gefahrvolle[], schicksalshafte[] Naturraum" (GS III, 93), der zuerst erlebt und schließlich geistig bezwungen worden sei. Seine eigene Version der Geistesgeschichte wird den, gefahrvollen, schicksalshaften Raum der Geschichte' in den Blick nehmen: seine mythische Schicksalshaftigkeit, die direkt hinein in den Abgrund führt.

\section{Das ,prekäre' Scheinen der Form}

Dieses Buch, das sich als Geschenkbuch wie kaum ein anderes eignet, kommt zur rechten Zeit. [...] Und während sonst, in anderen Sammlungen, die verbindenden Worte des Herausgebers meistens eine Plage sind, bedeuten in dieser Briefsammlung die zu jedem Brief gegebenen Einleitungen von Detlef $\mathrm{Holz}$ eine besondere Bereicherung: hier spricht ein Mann, den ein reiches Wissen nicht pedantisch, sondern weise gemacht hat. (Auerbach 1936: 447)

Was Erich Auerbach in der Schweizerischen National-Zeitung in einer Rezension als kommentierte Briefsammlung ankündigt, ist mehr als das: Es ist ein Requisitorium, ein ,Einspruch' gegen die Zeit. Vorher bereits, in den Jahren 1931/1932, hatte Benjamin die Briefe publiziert, siebenundzwanzig Texte insgesamt, einzeln jeweils, mit Kommentaren, in der Frankfurter Zeitung - ,Unter dem Strich'. ${ }^{6}$ Seiner Meinung nach wären erst hundert präsentabel gewesen. Schon dabei, während dieser Jahre, musste er den eigenen Namen verschweigen: der Faschismus hatte seine Schatten vorausgeworfen.

Benjamin hat sich in der Auswahl der Briefe auf den Zeitraum von 1783 bis 1883 beschränkt. Die große humanistische Epoche. Mit Goethe als Scheitel. Angefangen bei Georg Christoph Lichtenberg beschreibt Benjamin die Bewegung einer Epoche, in der das Bürgertum aus dem Windschatten der Feudalaristokratie heraustritt, seine

6 Die ausgewählten Briefe und Kommentare erscheinen im Feuilleton der Frankfurter Zeitung vom 1. April 1931 bis 31. Mai 1932. große Position bezieht, eine bürgerliche Intelligenz, von der aber - nach Goethes Tod - nur noch die Position übrigbleibt, nichts mehr der Geist, der sie ursprünglich trug. "Von Ehre ohne Ruhm, von Grösse ohne Glanz, von Würde ohne Sold", so ist das Buch untertitelt. Mit den Gründerjahren sieht Benjamin eine ganze Epoche ihrem Ende entgegengehen, ein Ende, das bereits Goethe vorausgesehen, ja, vorausgespürt hatte, sechs Jahre, bevor er starb. Eisenbahnen, Schnellposten, Dampfschiffe. Reichtum und Schnelligkeit, das ist es, was, jetzt' zählt:

Eigentlich ist es das Jahrhundert für die fähigen Köpfe, für leichtfassende praktische Menschen, die, mit einer gewissen Gewandtheit ausgestattet, ihre Superiorität über die Menge fühlen, wenn sie gleich selbst nicht zum Höchsten begabt sind. (Benjamin 1936, 10)

Eine solche Epoche, wie die vergangene, die kommt nicht wieder. Goethes Todesjahr halbiert den abgesteckten Zeitraum. Ursprünglich hatte Benjamin geplant, die Sammlung unter dem Titel "Das unterschlagene Deutschland. Briefe" herauszubringen. Es sollte ein anderes Deutschland repräsentieren, das bürgerlich-humanistische, in einer Zeit, in der der Geist bereits aus ihm verschwunden war. Das Exemplar, das er Siegfried Kracauer, seinem Freund und intellektuellen Weggefährten, zusandte, zeichnete er mit: „Für $\mathrm{S}$ Kracauer / diese Arche / die ich gebaut habe / als die faschistische Sintflut / zu steigen begann".?

Was unterscheidet Benjamins Sammlung in der methodischen Anlage von derjenigen Borchardts, auch und nicht zuletzt im Hinblick auf Goethe und dessen morphologisches Denken. Hierzu ist ein sogenannter "Nachtrag" zum "Fall Borchardt" aufschlussreich, den Benjamin verfasst hat und in dem es um den problematischen Scheincharakter seiner von Goethe entlehnten Formlehre geht:

Wenn die Aufgabe gestellt wäre, Borchardts Schrifttum aus jener eigentümlichen Erfahrung des Scheines heraus zu konstruieren, die kaum je so marktschreierisch, so verzweifelt aber auch kaum je so vollendet und ihrer Vollendung bewußt sich herausschrie, so hieße das zugleich ihn in Zusammenhänge einstel-

7 Neun Widmungen Benjamins in Exemplaren von Deutsche Menschen sind insgesamt überliefert, darunter auch an Adorno, Horkheimer und Gershom Scholem. Vgl. dazu Wizisla 2008. 
len, die alles andere als literarhistorische sind. Wer hätte man zu fragen - hat vor inm diese Erfahrung in solcher sinnlichen Bestimmtheit und Fülle gekannt. Man käme auf Hofmannsthal, eben darum sein großer Erzieher. Man stieße aber zuletzt auch auf Goethe. Und man deutet ein historisches Phänomen erstaunlicher Art an, mit der Behauptung, daß eben die Goethesche Eroberung einer Scheinwelt in Hofmannsthal und Rudolf Borchardt ihre erschreckend gelehrigen, erschreckend festgelegten Diadochen gehabt hat. Diese Goethesche Welt - unter denen des Meisters nur eine - ihnen die einzige, schiebt sich in einer Halluzination des Zeitsinns ihnen vor die wirkende und werdende; während sie selber allerdings wirkungs- und wandellos und darum nicht ohne Eignung ist, mit einer griechischen Vorwelt so verwechselt zu werden, wie Goethe es selber doch niemals getan hat. (GS VI, 201)

Benjamins Kritik an Borchardt setzt dort an, wo der Geist selber nur zu einem Erfüllungsgehilfen eines Werkes wird, das sich im Namen einer Idee an der sinnlichen Bestimmtheit und Fülle vergeht, als "Halluzination" vor die wirkende und werdende Welt schiebt. Der Schein herrscht, wo nicht mehr die Phänomene selbst sprechend zur Geltung kommen, dort, wo eine Gestalt alle anderen Gestalten dominiert. Dieses vermeintliche Verhängnis aber ist Benjamin angetreten mit seiner Briefanthologie, aufzusprengen': Im Moment des Untergangs, da die "faschistische Sintflut" zu steigen beginnt, legt Benjamin mit seiner Sammlung den Glutkern einer Hoffnung frei, die sich überhaupt erst gegen das, was sie verunmöglicht, als ,Eingedenken' bewähren kann. Nicht die großen Namen sind es, die seiner Anthologie ihren spezifischen Charakter als "Arche" verleihen, sondern die vielen Unbekannten, Vergessenen, das Latente: Johann Heinrich Kant, der an seinen Bruder Immanuel Kant, Friedrich Hölderlin, der an Casimir Ulrich Böhlendorf, Clemens Brentano, der an den Buchhändler Georg Andreas Reimer in Berlin schreibt:

„Denn wirken kann, im Reiche der Formen und als Form, d. h. also im strengsten Sinne des Wortes: unpolitisch, heute nur das durchaus Verborgene, Unscheinbare, das scheinbar, und bis zu einem gewissen Grade sogar wirklich, der Wirkung Entsagende." (GS VI, 202)

Die ,geistige Zugehörigkeit', die Borchardt über die Brüche der Tradition, das disparate Material der Geschichte hinweg restituieren zu können glaubte, bei Benjamin wird sie vollends fraglich, ,Heimat' damit prekär.
Borchardt und Benjamin sind in ihrer Wahlverwandtschaft und Gegensätzlichkeit Zeitgenossen in einem emphatischen Sinne. Ihre jeweiligen Zeitdiagnosen sind Ausdruck einer inneren Spannung der Epochenschwelle um 1900, die gerade aus der Differenz zwischen einem vermeintlich harmonisch geordneten Ganzen namens ,Natur' und dem ungemeinen Recht der Wirklichkeit erwächst. Wie man dabei mit dem ,Riss', der im 19. Jahrhundert so unheilbar durch die Zeit gefahren ist, umgeht, lässt sich wissenschaftstheoretisch als Konflikt zwischen Geistes- und Naturwissenschaften, zeitgeschichtlich als Konflikt zwischen Deutschland und Frankreich, geschichtsphilosophisch als Konflikt zwischen Tradition und Moderne, epistemologisch als Konflikt zwischen Anschauen und Denken rekonstruieren. Die Diskurse, die sich im Ausgang davon entwickeln, lassen die Frage in den Blick geraten, warum und in welcher Weise ,Natur' - verstanden als, geistige Heimat' - in einem tieferen Sinne um 1900 ,prekär' wird.

\section{Literaturverzeichnis}

\section{Quellen}

Adorno, Theodor W. (1974): Die Wunde Heine. In: ders.: Noten zur Literatur. Gesammelte Schriften, Bd. 11, Frankfurt a. M.: Suhrkamp. S. 95-100.

Auerbach, Erich (1936): Rezension zu Deutsche Menschen. In: National-Zeitung (Basel), 6. Dezember 1936 (Bücherseite). Wiederabgedruckt in: Momme Broderson (Hg.): Walter Benjamin. Werke und Nachlaß. Kritische Gesamtausgabe, Bd. 10, Frankfurt a. M. 2008: Suhrkamp, S. 447.

Benjamin, Walter (1924): Goethes Wahlverwandtschaften. In: Tiedermann, Rolf/Schweppenhäuser, Hermann (Hgg.) u. Mitw. v. Adorno, Theodor W./Scholem, Gershom: Walter Benjamin: Gesammelte Schriften. Bd. I.1, Frankfurt a. M.: Suhrkamp 1980, S. 123-201.

Benjamin, Walter (1927): Der Deutsche in der Landschaft. Besorgt von Rudolf Borchardt. In: Tiedermann, Rolf/Schweppenhäuser, Hermann (Hgg.) u. Mitw. v. Adorno, Theodor W./Scholem, Gershom: Walter Benjamin: Gesammelte Schriften. Bd. III, Frankfurt a. M.: Suhrkamp 1982, S. 91-94.

Benjamin, Walter (1936): Deutsche Menschen. In: Momme Broderson (Hg.): Walter Benjamin. Werke und Nachlaß. Kritische Gesamtausgabe, Bd. 10, Frankfurt a. M. 2008: Suhrkamp.

Benjamin, Walter (o. D.): "Zum Fall Borchardt diesen Nachtrag". In: Tiedermann, Rolf/Schweppenhäuser, Hermann (Hgg.) u. Mitw. v. Adorno, Theodor W./ 
Scholem, Gershom Walter Benjamin: Gesammelte Schriften. Bd. VI, Frankfurt a. M.: Suhrkamp 1985, S. 201-202.

Borchardt, Rudolf ([1907] 1920): Villa. In: ders.: Schriften. Prosa I, Berlin: Rowohlt, S. 5-44.

Borchardt, Rudolf ([1927] 2018): Der Deutsche in der Landschaft. In: Schalansky, Judith (Hg.): Naturkunden, 42, mit einem Nachw. v. Franck Hofmann. Berlin: Matthes \& Seitz.

Rudolf Borchardt ([1927] 1955): Schöpferische Restauration. In: Borchardt, Marie Luise ( $\mathrm{Hg}$.$) : Rudolf$ Borchardt: Reden. Stuttgart: Klett-Cotta, S. 230-253.

Willy Haas: Zwei Dokumente wider Willen. In: Die Literarische Welt. Berlin. Jg. 4, Nr. 5 vom 3. Februar 1928 , S. 5.

\section{Forschungsliteratur}

Hofmann, Franck (2018): Die Frage nach dem Menschen. Postscript. Nachwort zur Neuausgabe von Rudolf Borchardt: Der Deutsche in der Landschaft, Berlin: Matthes \& Seitz, S. 512-534

Höfer, Hannes (2015): Deutscher Universalismus. Zur mythologisierenden Konstruktion des Nationalen in der Literatur um 1800. Heidelberg: Winter.

Kauffmann, Kai (2003): Rudolf Borchardt und der ,Untergang der deutschen Nation'. Selbstinszenierung und Geschichtskonstruktion im essayistischen Werk. Tübingen: Niemeyer.
Korff, Hermann August (1923): Geist der Goethezeit. Versuch einer ideellen Entwicklung der klassischromantischen Literaturgeschichte, 3 Bde., Bd. 1. Leipzig: Weber.

Matz, Wolfgang (2011): Eine Kugel im Leibe. Walter Benjamin und Rudolf Borchardt: Judentum und deutsche Poesie. Göttingen: Wallstein.

Schmidt, Georg (2004): Staat, Nation und Universalismus: Weimar-Jena als Zentrum deutscher Identitätssuche im späten Alten Reich. In: Fink, Gonthier-Louis/Klinger, Andreas (Hgg.): Identitäten. Erfahrungen und Fiktionen um 1800, Frankfurt am Main u.a.: Peter Lang, S. 33-70

Steiger, Meike (2004): Von der Tonscherbe zur großen Erzählung. Zur Frage nach der Modernität von Rudolf Borchardts Geschichtsschreibung. In: Kauffmann, Kai (Hg.): Das wilde Fleisch der Zeit. Rudolf Borchardts Kulturgeschichtsschreibung. Stuttgart: Klett-Cotta, S. 24-46.

Wizisla, Erdmut (2008): Widmungen für die ersten Leser. In: Hahn, Barbara/ders. (Hg.): Walter Benjamins Deutsche Menschen. Göttingen: Wallstein, S. 45-67. 